\section{Dengue hemorrhagic fever in a peripheral blood stem cell transplant recipient: the first case report}

\author{
Jirayu Visuthranukul, ${ }^{1,2}$ \\ Udomsak Bunworasate, ${ }^{3}$ \\ Panisinee Lawasut, ${ }^{3}$ \\ Chusana Suankratay' \\ 'Division of Infectious Diseases, \\ Department of Medicine, Faculty of \\ Medicine, Chulalongkorn University, \\ Bangkok, Thailand; 'Division of Infectious \\ Diseases, Department of Medicine, Police \\ General Hospital, Bangkok, Thailand; \\ ${ }^{3}$ Division of Hematology, Department of \\ Medicine, Faculty of Medicine, \\ Chulalongkorn University, Bangkok, \\ Thailand
}

\section{Abstract}

Dengue infection, a mosquito-borne infectious disease in tropical and subtropical areas, has recently become an emerging global disease. The clinical course of dengue infection may be unfavorable in immunocompromised patients. In this report, we present a 16-year old female patient with acute myeloid leukemia who received allogeneic peripheral blood stem cell transplant five months prior to presentation. She was hospitalized at King Chulalongkorn Memorial Hospital, Bangkok, Thailand, due to fever, headache, and myalgia for one day. During hospitalization, she developed capillary leakage syndrome and progressive thrombocytopenia. A diagnosis of dengue hemorrhagic fever was made and confirmed by positive dengue serology and polymerase chain reaction testing. She made a full recovery 14 days after hospitalization. Our case possibly acquired dengue virus from infected mosquitoes while visiting her relatives four days before her present illness. In conclusion, this is the first reported case of dengue hemorrhagic fever in a peripheral blood stem cell transplant recipient. In addition, we review all previous reports of dengue infection in organ transplant recipients.

\section{Introduction}

Dengue infection is an acute infectious disease caused by four dengue virus serotypes 1 , 2,3 , and $4 .{ }^{1.7}$ The principal vector is Aedes aegypti, a mosquito with worldwide distribution in many tropical and subtropical areas.
The clinical spectrum of dengue infection varies from asymptomatic to severe disease. All serotypes produce a similar clinical illness characterized by acute fever, headache, generalized myalgia, nausea, and vomiting, and induce a life-long immunity that is specific to the infecting serotype. ${ }^{7.8}$ A small proportion of infected individuals may develop a severe form of disease, dengue hemorrhagic fever (DHF), characterized by fever, thrombocytopenia, hemorrhagic manifestations, and excessive capillary leakage probably leading to dengue shock syndrome (DSS) and death. ${ }^{1-7}$ The clinical course of dengue infection may be unfavorable in immunocompromised patients. Bone marrow transplant recipients have an impaired cell-mediated immunity, placing them at increased risk of infections. We report a case of DHF in a peripheral blood stem cell recipient, and review all previous reports of dengue infection in organ transplant recipients.

\section{Case Report}

A 16-year old female was hospitalized at King Chulalongkorn Memorial Hospital, Bangkok, Thailand, due to a high-grade fever without chills, bitemporal headache, generalized myalgia, and nausea one day prior to admission. She had returned four days before from visiting her relatives at Chon Buri, East Thailand. The patient had been diagnosed with acute myeloid leukemia (type M4) 14 months prior to the present illness when she noted acute fever, petechial rash, and bleeding of her gums and nose. She received induction and a consolidation course of chemotherapy. Allogeneic peripheral blood stem cell transplantation was performed five months before the present illness. She was in complete remission when last seen one month prior to her present illness. Complete blood count (CBC) showed hematocrit of $28 \%$, white blood cell count of $4.89 \times 10^{9} / \mathrm{L}$ (neutrophil $46 \%$, lymphocytes $40 \%$, and monocytes $6.9 \%$ ), and platelet count of $186 \times 10^{9} / \mathrm{L}$. Her current medications include cyclosporine, acyclovir, and cotrimoxazole. Physical examination revealed an acutely ill patient with body temperature of $39.7^{\circ} \mathrm{C}$ and bilateral anterior cervical lymphadenopathy. CBC showed hematocrit of $33 \%$, white blood cell count of $6.3 \times 10^{9} / \mathrm{L}$ (neutrophil $80 \%$, lymphocyte $8 \%$, atypical lymphocyte $5 \%$, and monocyte $7 \%$ ), and platelet count of $120 \times 10^{9} / \mathrm{L}$. Three days after hospitalization, a relapse of acute myeloid leukemia could not be excluded, and hence bone marrow was examined and revealed decreased cellularity, adequate megakaryocytes, increased histiocytes and eosinophils, consistent with reactive marrow to probable certain infection.
Correspondence: Chusana Suankratay, Division of Infectious Diseases, Department of Medicine, Chulalongkorn University, Bangkok 10330, Thailand. E-mail: chusana.s@chula.ac.th

Key words: dengue, dengue hemorrhagic fever, stem cell transplantation, bone marrow transplantation.

Conflict of interest: the authors reported no potential conflicts of interests.

Received for publication: 23 September 2009.

Revision received: 26 October 2009.

Accepted for publication: 27 October 2009.

This work is licensed under a Creative Commons Attribution 3.0 License (by-nc 3.0).

@C Copyright J. Visuthranukul et al., 2009

Infectious Disease Reports 2009; 1:e3 doi:10.4081/idr.2009.e3

Eight days after hospitalization, she noted petechial rash over both her legs, and physical examination revealed moderate hepatomegaly and right pleural effusion. CBC showed hematocrit of $38 \%$, white blood cell count of $8.84 \times 10 \%$ (neutrophil $79 \%$, lymphocyte $5 \%$, atypical lymphocyte $13 \%$, and monocyte $3 \%$ ), and platelet count of $16 \times 10^{\circ} / \mathrm{L}$. DHF was suspected, and later confirmed by enzyme-linked immunoassorbant assay ${ }^{9}$ and reverse transcriptase-polymerase chain reaction (PCR) testing. ${ }^{10}$ A diagnosis of primary dengue infection was made with dengue IgM of more than $40 \mathrm{U}$ and $\operatorname{Ig} \mathrm{M} / \operatorname{lgG}$ ratio of or more than 1.8:1 (dengue IgM rose from 86.65 to $121.03 \mathrm{U}$, and IgG rose from 49.18 to $134.01 \mathrm{U}$ ). Twelve days after hospitalization, she developed convalescent rash over her extremities. She eventually made a full recovery, and was discharged 14 days after hospitalization.

\section{Discussion}

This is the first reported case of dengue hemorrhagic fever in a peripheral blood stem cell transplant recipient. Our case had primary dengue infection, and possibly acquired dengue virus from infected mosquitoes while visiting her relatives at Chon Buri. Clinical manifestations of dengue infection in immunocompromised patients are usually similar to those noted in immunocompetent individuals. However, some patients experienced a longer duration (more than seven days) of illness than that in healthy individuals (4-7 days).1.3 A 23-year old renal transplant recipient developed dengue hemorrhagic fever which lasted for 19 days. ${ }^{11}$ Our patient also had a 12-day course of dengue hemorrhagic fever. 
In addition, an unusual presentation of dengue infection has been reported..$^{12,13}$ A 25 -year old patient with aplastic anemia with dengue infection developed polyserositis while he underwent conditioning for allogeneic stem cell transplant from his brother. ${ }^{12}$ Another case of unusual dengue infection was described in a renal transplant recipient who developed acute colitis one week after returning from Southeast Asia. $^{13}$

Furthermore, a transmission of dengue infection from an organ transplant donor during transplantation has been reported. ${ }^{11,14}$ One case of possible transmission of dengue infection from a living donor to a renal transplant recipient was described. ${ }^{11}$ This patient developed acute fever five days after receiving a kidney transplantation from his mother who later had a positive PCR test in the blood for dengue virus serotype 1 . Another case of dengue infection transmission was described in a bone marrow transplant recipient during a dengue epidemic in Puerto Rico in 1994. ${ }^{14}$ A 6-year old recipient developed an acute febrile episode four days after a transplant, and eventually died. Dengue serotype 4 was isolated from the blood and post-mortem tissues. The donor developed a dengue-like illness two days after a transplant. A diagnosis of dengue infection was confirmed by a positive dengue IgM from ELISA testing.

There are a few studies on the histopathology of bone marrow in dengue infection, and most of them are from post-mortem cases with severe dengue infection. ${ }^{15-17}$ Diffuse hypocellularity with and without hemophagocytosis is always described in bone marrow of patients with dengue hemorrhagic fever. In our case, examination of bone marrow revealed diffuse hypocellularity despite being performed early in the course of dengue infection.

This is the first report describing dengue hemorrhagic fever in a peripheral blood stem cell recipient. A high index of suspicion of dengue infection should be given to every transplant patient living or returning from endemic areas who presents with an acute fever in association with viral syndrome.

\section{References}

1. Rigau-Pérez JG, Clark GG, Gubler DJ, et al. Dengue and dengue haemorrhagic fever. Lancet 1998;352:971-7.

2. Jelinek T. Dengue fever in international travelers. Clin Infect Dis 2000;31:144-7.

3. Mairuhu AT, Mac Gillavry MR, Setiati TE, et al. Is clinical outcome of dengue-virus infections influenced by coagulation and fibrinolysis? A critical review of the evidence. Lancet Infect Dis 2003;3:33-41.

4. Gibbons RV, Vaughn DW. Dengue: an escalating problem. Br Med J 2002;324:1563-6.

5. Guzmán MG, Kourí G. Dengue: an update. Lancet Infect Dis 2002;2:33-42.

6. Wilder-Smith A, Schwartz E. Dengue in travelers. N Engl J Med 2005;353:924-32.

7. Halstead SB. Dengue. Lancet 2007;370: 1644-52.

8. Lee MS, Hwang KP, Chen TC, et al. Clinical characteristics of dengue and dengue hemorrhagic fever in a medical center of southern Taiwan during the 2002 epidemic. J Microbiol Immunol Infect 2006;39: 121-9.

9. Vaughn DW, Green S, Kalayanarooj S, et al.
Dengue in the early febrile phase: viremia and antibody responses. J Infect Dis 1997; 176:322-30.

10. Lanciotti RS, Calisher CH, Gubler DJ, et al. Rapid detection and typing of dengue viruses from clinical samples by using reverse transcriptase-polymerase chain reaction. J Clin Microbiol 1992;30:545-51.

11. Tan FL, Loh DL, Prabhakaran K, et al. Dengue haemorrhagic fever after living donor renal transplantation. Nephrol Dial Transplant 2005;20:447-8.

12. Ullah K, Ahmed P, Raza S, et al. Allogeneic stem cell transplantation in hematological disorders: single center experience from Pakistan. Transplant Proc 2007;39:334757.

13. Park SB, Ryu SY, Jin KB, et al. Acute colitis associated with dengue fever in a renal transplant recipient. Transplant Proc 2008; 40:2431-2.

14. Rigau-Pérez JG, Vorndam AV, Clark GG. The dengue and dengue hemorrhagic fever epidemic in Puerto Rico, 1994-1995. Am J Trop Med Hyg 2001;64,67-74.

15. Peres LC, Saggioro FP, Dias LB Jr, et al. Infectious diseases in paediatric pathology: experience from a developing country. Pathology 2008;40:161-75.

16. Rothwell SW, Putnak R, La Russa VF. Dengue-2 virus infection of human bone marrow: characterization of dengue-2 antigen-positive stromal cells. Am J Trop Med Hyg 1996;54:503-10.

17. Jain D, Singh T. Dengue virus related hemophagocytosis: a rare case report. Hematology 2008;13:286-8. 